\title{
Editorial: Signal Processing for Communication/Biomedical Systems and Reliability Improvement
}

\author{
Ching-Te Chiu • Lei Wang • Yin-Tsung Hwang
}

Published online: 25 December 2014

(C) Springer Science+Business Media New York 2014

Signal Processing plays an important role in communications, biomedical/multimedia systems, and semiconductor devices. With the continuous scaling of semiconductor technologies, key advanced techniques have to be developed in SP algorithms, architectures, design methods, and prototyping to meet the new requirements and improve reliability. This issue is focused on signal processing techniques for communications/biomedical systems and reliability improvement under technology scaling. Several case studies are given in various applications. It shows the impact of these signal processing technologies on the performance of a system.

This special issue consists of eight papers. The first three papers are case studies of signal processing technologies for communication systems. They showcase how to improve the system performance by employing the parallelism of GPU processors. The fourth and fifth papers address the challenges and techniques for improving reliability under

\section{C.-T. Chiu ( $\square)$}

Department of Computer Science and Institute

of Communications Engineering, National Tsing

Hua University, Hsinchu, Taiwan

e-mail: ctchiu@cs.nthu.edu.tw

\section{Wang}

Department of Electrical and Computer Engineering,

University of Connecticut, Storrs, CT, USA

e-mail: leiwang@engr.uconn.edu

\section{Y.-T. Hwang}

Department of Electrical Engineering,

National Chung Hsing University,

Taichung, Taiwan

e-mail: hwangyt@dragon.nchu.edu.tw technology scaling. The last three papers present efficient algorithms and designs for biomedical and multimedia systems. They are brief described below.

The first paper, Opportunities and Challenges of Digital Signal Processing in Deeply Technology-Scaled Transceivers (doi:10.1007/s11265-014-0940-x), presents several transceiver examples to depict the signal processing opportunities and challenges on recent and ongoing design activities. For the parameter estimation, calibration and compensation of reconfigurable transceivers, there is a need to have flexible processors on chip running versatile algorithms that can even perform model identification by themselves. The high performance signal processing in signal paths also calls for disruptive solutions due to the high duty cycle, high sampling rate and stringent power/area constraints. Aggressive algorithm/architecture optimizations, analog driven digital design techniques and stochastic computation techniques are considered to be promising. The second paper, GPU Accelerated Belief Propagation Decoding of Non-Binary LDPC Codes with Parallel and Sequential Scheduling (doi:10.1007/s11265-014-0927-7), presents an efficient implementation of a signed-log domain FFT decoder for non-binary irregular LDPC codes, which exploits the inherent massive parallelization capabilities of message passing decoders. The Nvidia's Compute Unified Device Architecture (CUDA) is employed to incorporate the available processing power of state-of-the art Graphics Processing Units (GPUs). Furthermore, a CUDA implementation of the signed-log domain FFT decoder using the so-called layered up date rule is presented. The third paper, Using graphics processing units in an LTE base station (doi:10.1007/s11265-014-0932-x), develops parallel implementations of key kernels to evaluate the merits of using 
GPUs as the baseband signal processor. The mapping of key kernels onto a multi-GPU system minimizing the number of required GPUs and the overall subframe processing latency is studied. Their results show that the baseband subsystem in an LTE base station can support up to $150 \mathrm{Mbps}$ peak data rate using four NVIDIA GTX 680 GPUs and commercial motherboards.

The fourth paper, Hardware Specialization in Lowpower Sensing Applications to Address Energy and Resilience (doi:10.1007/s11265-014-0931-y), explores implications of introducing machine learning capabilities within a hardware-specialized platform for low power embedded sensing applications. Such a platform is useful for algorithms well suited for analyzing complex sensor signals under strict energy constraints. However, the benefits go further, enabling the effects of errors to be overcome in the presence of hardware faults within the platform. Although errors can result in substantial bit-level perturbations, the approach described views these as an alteration on the way that information is encoded within the embedded data. The new information encoding can thus be learned in the form of an error-aware model. The fifth paper, Low Energy Signal Processing Techniques for Reliability Improvement of High-Density NAND Flash Memory (doi:10.1007/s11265-014-0943-7), presents signal processing and error correction techniques that can overcome the reliability problem while minimizing the energy consumption. These techniques include efficient estimation of the threshold voltage distribution, CCI cancellation aware soft-information computation, and low-energy soft-decision error correction.

The sixth paper, High Frame Rate 3-D Ultrasound Imaging Using Separable Beamforming (doi:10.1007/ s11265-014-0933-9), presents a separable beamforming procedure for both 3-D synthetic aperture and plane wave systems that drastically reduces computational and hence power requirements. The proposed method is based on a separable delay decomposition method that minimizes phase error. The proposed separable synthetic aperture system achieves 19-fold complexity reduction and the proposed plane wave separable system achieves 12fold complexity reduction compared to the corresponding non-separable beamforming baseline systems. The performance of the fixed-point-precision separable beamforming and iterative delay calculation through Field II simulations is also verified. The seventh paper, An intelligent digital microfluidic processor for biomedical detection (doi:10.1007/s11265-014-0939-3), demonstrates a novel prototype processor that integrates the functions of microfluidic actuation, droplet location readback, and high sensitivity measurement window for personalized medicine. This potential architecture solves lots of traditional development bottlenecks and features easy-to-control, easy-tomonitor, system automation and high accuracy for bioassay detection purposes. In the last paper, Edge Curve Scaling and Smoothing with Cubic Spline Interpolation for Image Up-Scaling (doi:10.1007/s11265-014-0936-6), an efficient image upscaling method is presented to recover high frequency components of contour regions to improve the sharpness and reduce ringing artifacts. To reduce the computation cost, the patch-based method is applied only to the up-scaled edge map to recover the high frequency components. Experimental results show the proposed method can effectively preserve the image quality with a significant reduction on the execution time.

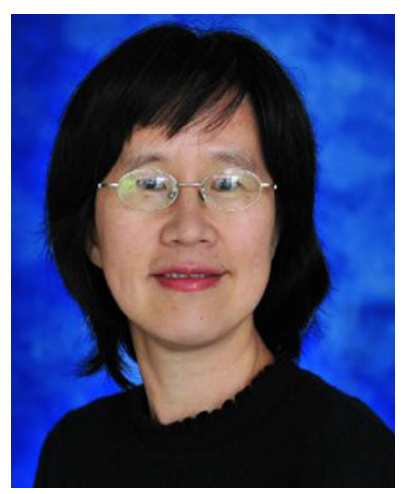

Ching- Te Chiu received her B.S. and M.S. degrees from National Taiwan University, Taipei, Taiwan. She received her Ph.D. degree from University of Maryland, College Park, Maryland, USA, all in electrical engineering. She was an Associate Professor with National Chung Cheng University, ChiaYi, Taiwan. She was member of technical staff with AT\&T, Murry Hill, New Jersey, and at Lucent Technologies, Murry Hill, New Jersey, and with Agere Systems. She is currently a Professor at the Computer Science Department and Institute of Communications Engineering, National Tsing Hua University, Hsinchu, Taiwan. Her research interests include High Dynamic Range Image and Video Processing, Super Resolution, Pattern Recognition, High Speed SerDes design, Multi-chip Interconnect, and Fault Tolerance for Network-on-Chip design. Dr. Chiu won the first prize award, the best advisor award, and the best innovation award of the Golden Silicon Award in 2006. She serves as a TC member of the IEEE Circuits and Systems Society, Nanoelectronics and Gigascale Systems Group, and the IEEE Signal Processing Society, Design and Implementation of Signal Processing Systems group. She is the program chair of the first IEEE Signal Processing Society Summer School at Hsinchu, Taiwan 2011 and technical program chair of IEEE workshop on signal processing system (SiPS) 2013. She serves as associate editor of IEEE Transactions on Circuits and Systems I and Journal of Signal Processing Systems. 


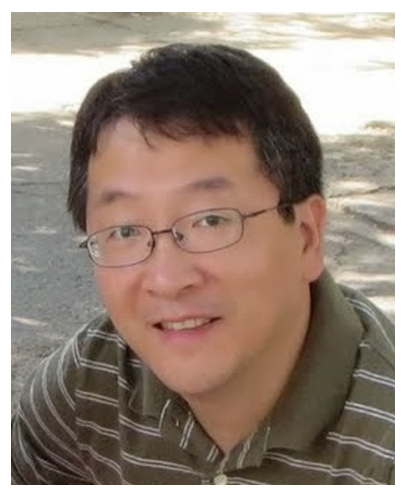

Lei Wang received the B.S. degree and the M.S. degree from Tsinghua University, China, in 1992 and 1996, respectively, and the Ph.D. degree from the University of Illinois at Urbana-Champaign in 2001. During the summer of 1999, Dr. Wang worked at Microprocessor Research Laboratories, Intel Corporation, in Hillsboro, OR, where his work involved development of high-speed and noise-tolerant VLSI circuits and design methodologies.

From December 2001 to July 2004, he was with Microprocessor Technology Laboratories, Hewlett-Packard Company, in Fort Collins, $\mathrm{CO}$, where he participated in the design of the first dual-core multithreaded Itanium Architecture Processor, a joint project between Intel and Hewlett-Packard. Since August 2004, he has been with the Department of Electrical and Computer Engineering, University of Connecticut, where he is currently a Francis L. Castleman Associate Professor. Dr. Wang is a recipient of the National Science Foundation CAREER Award. He is a member of IEEE Signal Processing Society Technical Committee on Design and Implementation of Signal Processing Systems. He has been serving as an Associate Editor for the IEEE Transactions on Computers since 2010, Senior Area Editor for the IEEE Signal Processing Letters since 2014, and on the Steering Committee of the IEEE Transactions on Multi-Scale Computing Systems. He served as an Associate Editor for the IEEE Signal Processing Letters from 2012 to 2014. He also served as a Guest Editor for the IEEE Transactions on Emerging Topics in Computing and a Guest Editor for the Springer Journal of Signal Processing Systems. Dr. Wang is a Senior Member of IEEE.

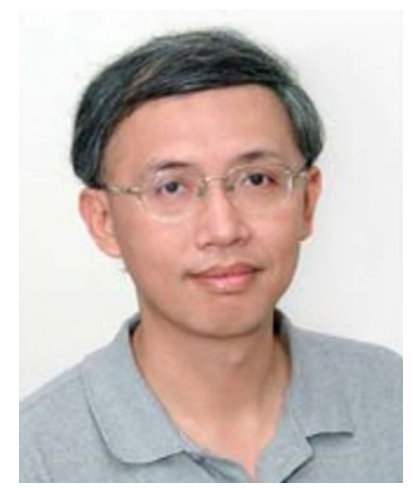

Yin- Tsung Hwang obtained his BS and MS degrees, both in electronic engineering, from National Chiao Tung University, Hsinchu, Taiwan, in 1983 and 1985, respectively. He received the Ph.D. degree from the department of Electrical \& Computer Engineering, University of Wisconsin, Madison in 1993. He then became a faculty member of the Department of Electronic Engineering, National Yunlin University of Science \& Technology in Taiwan. In 2004, he joined the department of Electrical Engineering, National Chung Hsing University and is now a professor. His research interests include VLSI designs for wireless base band processing, video/image signal processing, and low power VLSI circuit designs. 